\title{
Low-frequency noise in nFinFETs of different dimensions processed in strained and non-strained SOI wafers
}

\author{
N. Lukyanchikova ${ }^{1}$, N. Garbar ${ }^{1}$, V. Kudina ${ }^{1}$, A. Smolanka ${ }^{1}$, E. Simoen ${ }^{2}$ and C. Claeys ${ }^{2,3}$ \\ ${ }^{1} V$. Lashkaryov Institute of Semiconductor Physics, NAS of Ukraine \\ 45, prospect Nauky, 03028 Kyiv, Ukraine \\ Phone: +380445256453; e-mail: natali@isp.kiev.ua, kudinavaleriya@yahoo.com \\ ${ }^{2}$ IMEC,75, Kapeldreef, B-3001 Leuven, Belgium \\ ${ }^{3}$ KU Leuven, 10, Kasteelpark Arenberg, B-3001 Leuven, Belgium \\ E-mail: simoen@imec.be, claeys@imec.be
}

\begin{abstract}
The results of low-frequency noise investigation in fully-depleted (FD) nFinFETs of $W_{\text {eff }}=0.02$ to $9.87 \mu \mathrm{m}, L_{\text {eff }}=0.06$ to $9.9 \mu \mathrm{m}$, processed on standard (SOI) and strained (sSOI) wafers are presented. It is shown that the McWhorter noise is typical at zero back gate voltage for the devices studied and the density of the corresponding noisy traps in the $\mathrm{SiO}_{2}$ portion of the gate oxide is, as a rule, much higher than that in the $\mathrm{HfO}_{2}$ portion. The results on the McWhorter noise are used for studying the behavior of the electron mobility $\mu$ and the free electron density $N_{S}$ in the channel at $V^{*} \geq 0.4 \mathrm{~V}$ where $V^{*}$ is the gate overdrive voltage. It is also shown that the Linear Kink Effect (LKE) Lorentzians appear in the low-frequency noise spectra at an accumulation back gate voltage and that the parameters of those Lorentzians are different for the sSOI and SOI nFinFETs. This is the first observation of the LKE noise under a back-gate accumulation bias for sufficiently wide nMuGFET.
\end{abstract}

Keywords: low-frequency noise, FinFET, fully-depleted, SOI, sSOI, Linear Kink Effect.

Manuscript received 09.06.08; accepted for publication 20.06.08; published online 15.09.08.

\section{Introduction}

In order to overcome the current limitations of traditional dimensional scaling, performance boosters like strain and multiple gate architectures are currently of strong interest. The combination of both by fabricating for example n-channel FinFETs on strainedSilicon-on-Insulator (sSOI) substrates may yield additional drive current improvement compared with standard SOI substrates. While this is aiming in the first place to digital applications, the question arises what the analog potential of such technologies is. An important aspect there is the low-frequency (LF) noise behavior. The fact that the sidewall channels are on (110) faces for a standard (100) SOI substrate raises concerns regarding a higher density of interface and bulk oxide traps, and, hence, the LF noise. Another concern may be the application of strain on the quality of the gate oxide/silicon interface, which can be addressed by noise measurements. It is the aim of the present work to investigate the noise in n-channel triple gate MuGFETs with a $\mathrm{SiO}_{2} / \mathrm{HfO}_{2} / \mathrm{TiN}$ gate stack, fabricated on sSOI and SOI substrates, as a function of the device dimensions and bias conditions. It is shown that for most devices studied, a higher trap density is derived in the vicinity of the $\mathrm{SiO}_{2} / \mathrm{HfO}_{2}$ interface compared with the bulk $\mathrm{HfO}_{2}$. Only a minor impact of the strain has been noted. In addition and maybe more of academic value is the first observation of the Linear Kink Effect (LKE) noise under a back-gate accumulation bias for sufficiently wide nMuGFETs. In this case, a different behavior is found between SOI and sSOI devices.

\section{Experimental}

The investigated devices were n-channel fully-depleted (FD) FinFETs processed on both the standard (SOI) and strained (sSOI) SOI wafers. The parameters of the devices were as follows: $h=65 \mathrm{~nm}$ and $55 \mathrm{~nm}$ for SOI and sSOI nFinFETs, respectively, $W_{\text {eff }}=0.02$ to $9.87 \mu \mathrm{m}, L_{\text {eff }}=0.06$ to $9.9 \mathrm{~m}$, where $\mathrm{h}$ is the fin height, $W_{\text {eff }}$ and $L_{\text {eff }}$ are the effective fin width and length, respectively. In the case of $W_{\text {eff }}=0.02 \mu \mathrm{m}$ the multiple fin configuration was used where the fin number $N_{\text {fin }}$ was equal to 30 . The full device width $Z$ has been calculated by the formula $Z=N_{\text {fin }} \cdot\left(2 h+W_{\text {eff }}\right)$. The gate stack consisted of $2 \mathrm{~nm} \mathrm{HfO}_{2}$ on the top of $1 \mathrm{~nm}$ interfacial $\mathrm{SiO}_{2}$, so that one has for the equivalent oxide 
thickness: $t_{\mathrm{EOT}}=1.9 \mathrm{~nm}$. The gate electrode was $5 \mathrm{~nm}$ MOCVD TiN with a $100 \mathrm{~nm}$ poly-Si cap. No channel doping was used.

The drain current noise spectral density $S_{I}(f)$ within the frequency range $f=0.7 \mathrm{~Hz}$ to $100 \mathrm{kHz}$ was measured on wafer at $0.3 \mathrm{~V} \leq V_{\mathrm{GF}} \leq 1.6 \mathrm{~V}$, and $V_{\mathrm{DS}}=25 \mathrm{mV}$ for $V_{\mathrm{GB}}=0$ (an accumulation back-gate voltage) where $V_{\mathrm{GF}}$, $V_{\mathrm{DS}}$ and $V_{\mathrm{GB}}$ are the front gate, back gate and drain voltage, respectively.

\section{Results and discussions}

1. The families of the drain current noise spectra $S_{I}(f)$ measured at different $V_{\mathrm{GF}}$ and $V_{\mathrm{GB}}=0$ for the sSOI and relatively long SOI devices of $W_{\text {eff }}=0.02 \mu \mathrm{m}$ are shown in Fig. 1. It is seen that for the SOI FinFETs the noise spectra are of the $1 / f$ type up to sufficiently high frequencies where the $1 / f$ component is lost in the Nyquist noise. It is also seen that for the sSOI FinFETs the $1 / f$ portion of the noise spectra is observed only at $f>400 \mathrm{~Hz}$ while $S_{I} \sim(1 / f)^{0.7}$ takes place at $f<400 \mathrm{~Hz}$. Therefore, an essential difference in the shape of the noise spectra for the standard and strained devices of $W_{\text {eff }}=0.02 \mu \mathrm{m}$ shows itself at $f<400 \mathrm{~Hz}$. At the same time, it has been found that for the SOI FinFETs of $W_{\text {eff }} \geq 0.12 \mu \mathrm{m}$ the spectra similar to those shown in Fig. $1 \mathrm{~b}$ are typical. It should be noted that similar spectra are also observed for short $\left(L_{\text {eff }} \leq 0.16 \mu \mathrm{m}\right)$ SOI devices of $W_{\text {eff }}=0.02 \mu \mathrm{m}$.

Fig. 2 demonstrates the dependences of $S_{I}$ normalized for $L_{\text {eff }}$ and $Z$ on the gate overdrive voltage $V^{*}$ measured at $f=3 \mathrm{kHz}$ (curves 1 to 3 ) at which the $1 / f$ noise prevails for both the SSOI and SOI FinFETs and at $f=10 \mathrm{~Hz}$ (curves 4 and 5) that corresponds to the $(1 / f)^{0.7}$ noise. It is seen that $S_{I}$ does not depend on $V^{*}$ at $V^{*}>(0.2-0.8) \mathrm{V}$ for both the $1 / f$ and $(1 / f)^{0.7}$ noise components. It should be noted that such a behavior is typical for the low-frequency noise of the McWhorter type $[1,2]$.

It is known that the McWhorter model ascribes the noise to the fluctuations of the number of electrons in the channel accompanying the electron exchange between the channel and the slow traps located in the gate dielectric at various distances $x$ from the $\mathrm{Si} / \mathrm{SiO}_{2}$ interface. If those traps are distributed homogeneously over $x$, the $1 / f$ noise has to be observed. However, if the density of the noisy traps, $N_{\mathrm{ot}}$, decreases with increasing $x$, the noise spectrum has to be of the $(1 / \mathrm{f})^{m}$ shape where $m<1 \quad[1,3]$. Then the dependences $S_{I} \sim(1 / f)^{0.7}$ considered above and observed at $f<400 \mathrm{~Hz}$ can be explained by the decrease of $N_{\text {ot }}$ with increasing $x$ at $x>x_{0}$. The value of $x_{0}$ can be estimated using the formula $x_{0}=\lambda \ln \left[\left(2 \pi f_{0} \tau_{\min }\right)^{-1}\right]$ where $\lambda=0.1 \mathrm{~nm}$ is the tunneling parameter, $f_{0}=400 \mathrm{~Hz}$ and $\tau_{\min }=10^{-10} \mathrm{~s}$ [3], which gives $x_{0}=1.5 \mathrm{~nm}$. As to the $1 / f$ noise showing itself at $f>400 \mathrm{~Hz}$ in the sSOI devices, this noise corresponds to the traps located at $x<x_{0}$, i.e. more close to the $\mathrm{Si} / \mathrm{SiO}_{2}$ interface.
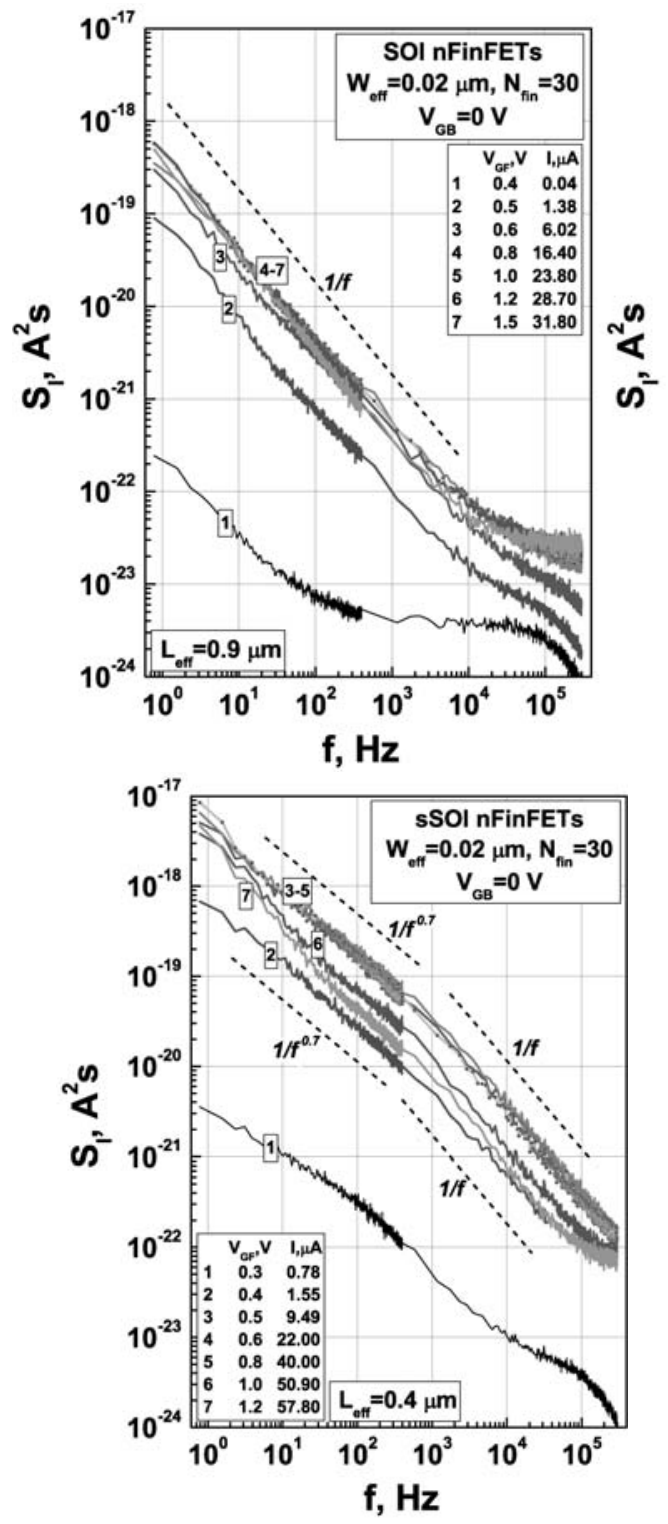

Fig. 1. Spectra of the drain current noise for the SOI (a) and sSOI (b) FD nFinFETs of $W_{\text {eff }}=0.02 \mu$ m measured at $V_{\mathrm{GB}}=0$.

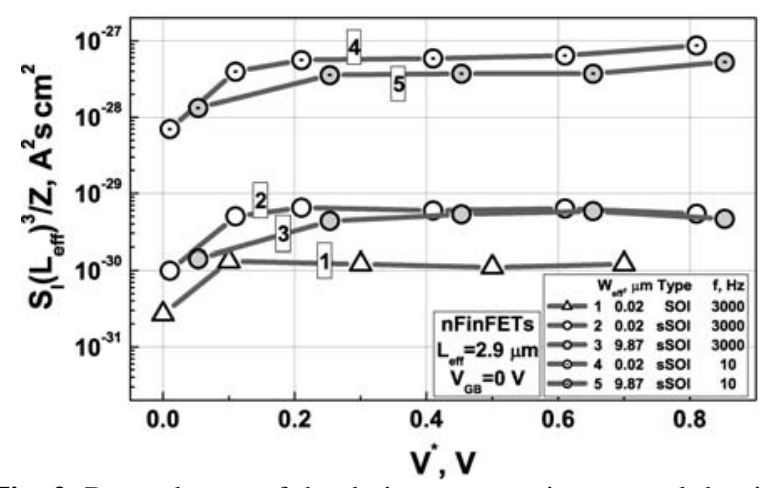

Fig. 2. Dependences of the drain current noise spectral density normalized for $L_{\text {eff }}$ and $Z$ on the gate overdrive voltage measured at $f=3 \mathrm{kHz}(1-3)$ and $10 \mathrm{~Hz}$ (4 and 5) for SOI (1) and sSOI (2-5) nFinFETs of $\mathrm{W}_{\text {eff }}=0.02 \mu \mathrm{m}(1,2$ and 4$)$ and $9.87 \mu \mathrm{m}(3$ and 5$) ; V_{\mathrm{GB}}=0$. 

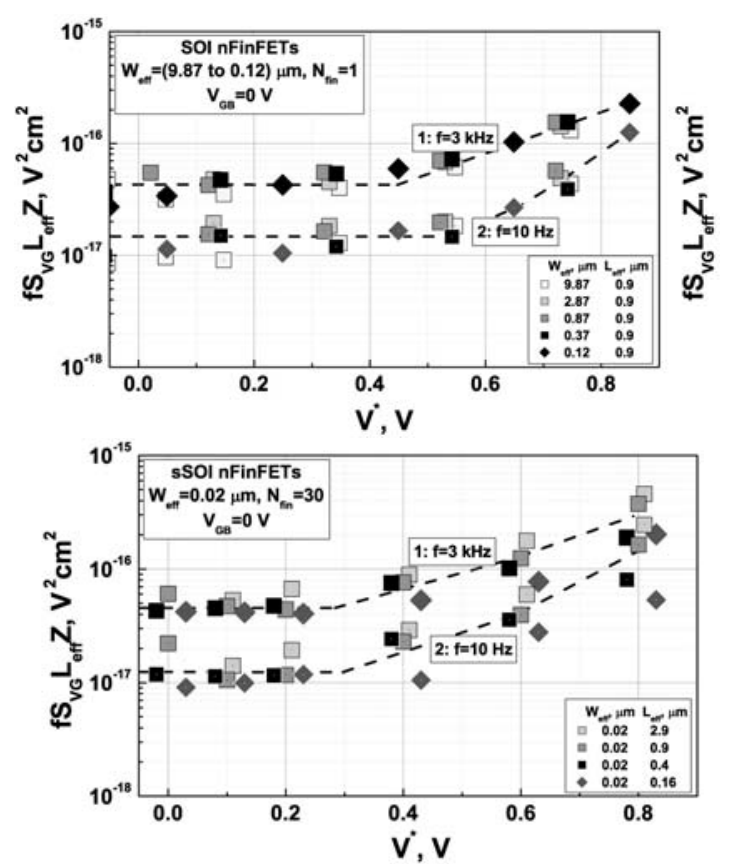

Fig. 3. Equivalent gate voltage noise normalized for $L_{\text {eff }}$ and $Z$ at different gate overdrive voltages for SOI nFinFETs of $W_{\text {eff }}=(0.12-9.87) \mu \mathrm{m} \quad$ and $\quad L_{\text {eff }}=0.9 \mu \mathrm{m}(\mathrm{a}) \quad$ and $\quad \mathrm{sSOI}$ nFinFETs of $W_{\text {eff }}=0.02 \mu \mathrm{m}$ and $L_{\text {eff }}=(0.16-2.9) \mu \mathrm{m}$ (b) measured at $f=3 \mathrm{kHz}(1)$ and $10 \mathrm{~Hz}(2) ; V_{\mathrm{GB}}=0$.

The dependences of the value of $S_{\mathrm{VG}} L_{\mathrm{eff}} Z$ on $V^{*}$ where $S_{\mathrm{VG}}$ is the spectral density of the equivalent gate voltage noise determined by $S_{\mathrm{VG}}=\left[S_{I} /\left(g_{m}\right)^{2}\right]$, where $g_{m}$ is the transconductance are shown in Fig. 3. As is seen, the typical for the McWhorter noise plateaus where $S_{\mathrm{VG}}$ is independent of the gate overdrive voltage manifest themselves in the range $0<V^{*} \leq 0.4 \mathrm{~V}$ for both $1 / f$ noise (curve 1$)$ and $(1 / f)^{0.7}$ noise (curve 2).

Fig. 4 presents the values of $N_{\mathrm{ot}}$ calculated by the formula $N_{\mathrm{ot}}=\left(f S_{\mathrm{VG}} L_{\text {eff }} Z C_{0}^{2}\right) / q^{2} k T \lambda$, where $S_{\mathrm{VG}}$ corresponds to the above mentioned plateau, $C_{0}$ is the capacitance of the gate oxide per $\mathrm{cm}^{2}, q$ is the electron charge, $k$ is the Boltzmann constant and $T$ is the temperature. The open circles and triangles in Fig. 4 correspond to the $1 / f$ noise component and relate to the traps located at $x<x_{0}$ in the sSOI FinFETs and in the SOI ones of some dimensions ( $W_{\text {eff }}=9.87 \mu \mathrm{m}$ or $W_{\text {eff }}=0.02 \mu \mathrm{m}$ and $L_{\text {eff }}=0.16 \mu \mathrm{m}$ ) as well as to the traps distributed homogeneously over $x$ in the SOI FinFETs of $W_{\text {eff }}=0.02 \mu \mathrm{m}$ and $L_{\text {eff }}>0.16 \mu \mathrm{m}$. The data shown in Fig. 4 by the dot center circles and triangles have been found by application of the formula $N_{\mathrm{ot}}=\left(f S_{\mathrm{VG}} L_{\mathrm{eff}} Z C_{0}^{2}\right) / q^{2} k T \lambda$ to the results measured for the $(1 / f)^{0.7}$ noise at $f=10 \mathrm{~Hz}$. Note that the frequency $f=10 \mathrm{~Hz}$ corresponds to $x=1.93 \mathrm{~nm}$.

It is seen from Fig. 4 that $N_{\text {ot }}=(2$ to 3$) \times 10^{19} \mathrm{~cm}^{-}$ ${ }^{3} \mathrm{eV}^{-1}$ at $x<x_{0} \approx 1.5 \mathrm{~nm}$ while the lower values of $N_{\text {ot }}$ $\left[N_{\mathrm{ot}}=(4\right.$ to 8$) \times 10^{18} \mathrm{~cm}^{-3} \mathrm{eV}^{-1}$ ] have been found at $x>x_{0}$ for the sSOI devices and the SOI ones of $W_{\text {eff }}=9.87 \mu \mathrm{m}$. It is also seen that the values of $N_{\mathrm{ot}}$ responsible for both

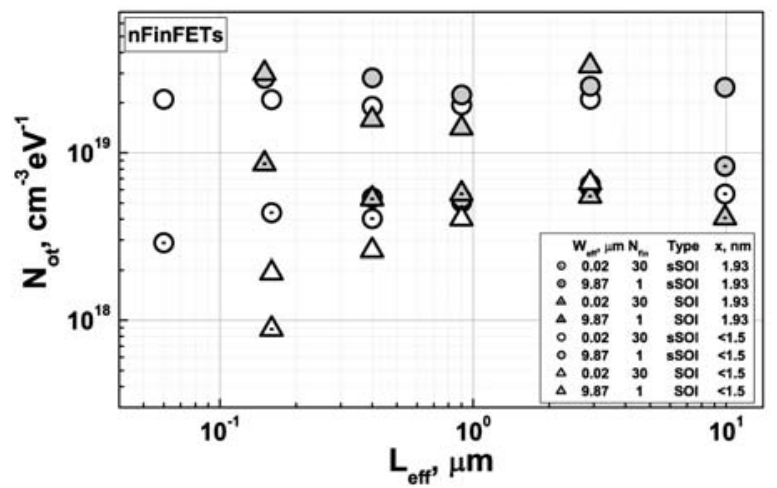

Fig. 4. Density of traps for SOI and sSOI nFinFETs of different $L_{\text {eff }}$ and $W_{\text {eff }}$ located in the gate oxide at various distances $x$ from the $\mathrm{SiO}_{2}$ interface.

the $1 / f$ and $(1 / f)^{0.7}$ noise in the devices of those types are practically independent of $W_{\text {eff }}$ and $L_{\text {eff. }}$ It should be noted that the estimated value of $x_{0}(1.5 \mathrm{~nm})$ is close to the thickness of the interfacial oxide layer $(1 \mathrm{~nm})$ used in the gate stack. This suggests that the traps characterized by the above mentioned higher density are located just in that oxide.

As to the SOI FinFETs of $W_{\text {eff }}=0.02 \mu \mathrm{m}$, it is seen from Fig. 4 that in the case where $L_{\text {eff }}>0.16 \mu \mathrm{m}$ and the traps are distributed homogeneously over $x$, the value of $N_{\text {ot }}$ decreases from $7 \times 10^{18} \mathrm{~cm}^{-3} \mathrm{eV}^{-1}$ to $3 \times 10^{18} \mathrm{~cm}^{-3} \mathrm{eV}^{-1}$ as far as $L_{\text {eff }}$ decreases from 2.9 to $0.4 \mu \mathrm{m}$. It is also seen from Fig. 4 that in the case where $L_{\text {eff }}=0.16 \mu \mathrm{m}$ and the values of $N_{\text {ot }}$ are different at $x<x_{0}$ and $x>x_{0}$, they appear to be relatively low, namely: $N_{\mathrm{ot}}=2 \times 10^{18} \mathrm{~cm}^{-}$ ${ }^{3} \mathrm{eV}^{-1}$ and $N_{\mathrm{ot}}=9 \times 10^{17} \mathrm{~cm}^{-3} \mathrm{eV}^{-1}$ for $x<x_{0}$ and $x>x_{0}$, respectively.

2. The obtained noise results can be used when considering the dimension behavior of the electron mobility $\mu$ as well as the behavior of the dependences $I\left(V^{*}\right)$ in the devices studied.

The values of $\mu_{m}$ corresponding to maximal values of the transconductance are shown in Fig. 5 for the devices of different types and dimensions. It is seen that: (i) $\mu_{m}$ decreases with decreasing $L_{\text {eff }}$ (Fig. $5 a$ ); (ii) at not too small $\mathrm{L}_{\text {eff }}$ the values of $\mu_{m}$ for the sSOI devices are higher than those for SOI (Fig. $5 \mathrm{a}$ and b); (iii) $\mu_{m}$ increases with decreasing $W_{\text {eff }}$ at $W_{\text {eff }}>0.9 \mu \mathrm{m}$ and becomes practically independent of $W_{\text {eff }}$ at $W_{\text {eff }}<0.9 \mu \mathrm{m}$ (Fig. 5b). Note that the same features have been observed previously in the $65 \mathrm{~nm}$ FD planar SOI nMOSFETs [4].

It is known that the decrease of $\mu_{m}$ with decreasing $L_{\text {eff }}$ can be connected with increasing $N_{\text {ot }}$ [5]. The increase of $N_{\mathrm{ot}}$ with increasing $W_{\text {eff }}$ could be also responsible for the corresponding decrease of $\mu_{m}$. However, a comparison of Fig. 5 with Fig. 4 shows that there is no correlation between $\mu_{m}\left(L_{\text {eff }}, W_{\text {eff }}\right)$ and $N_{\text {ot }}\left(L_{\text {eff }}, W_{\text {eff }}\right)$. Therefore, the dimension dependences of $\mu_{m}$ observed are not connected with the dimension dependences of $N_{\mathrm{ot}}$. 

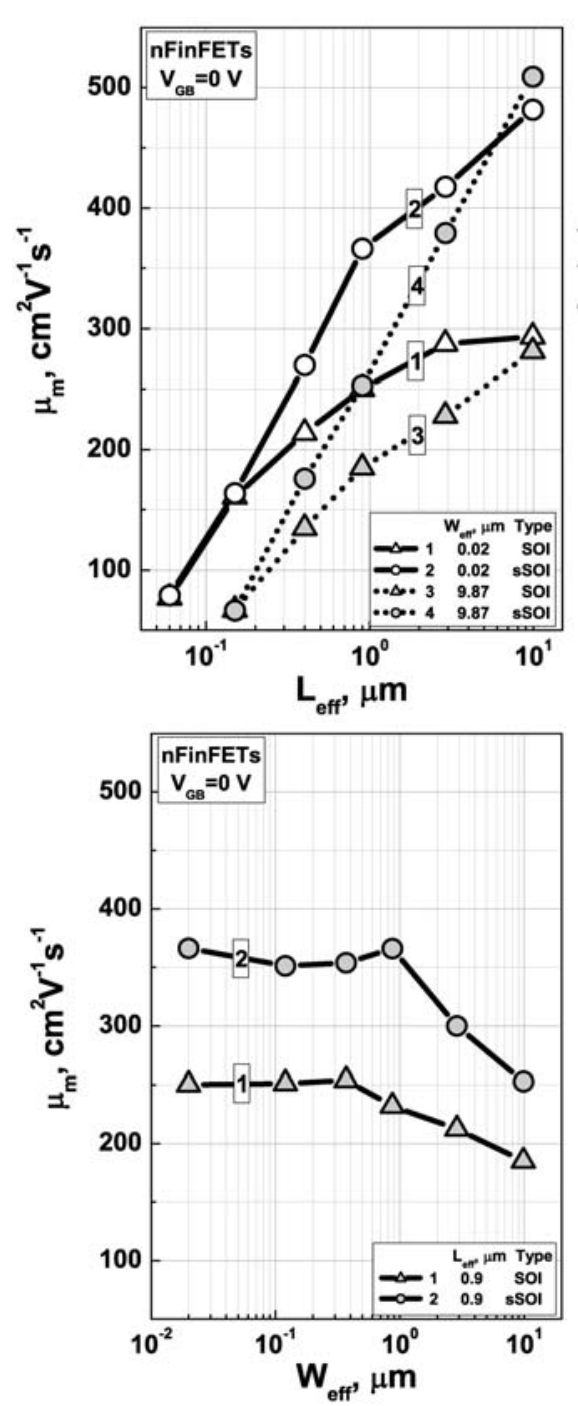

Fig. 5. Electron mobility corresponding to the maximum of the transconductance at different $L_{\text {eff }}$ (a) for SOI (1 and 3) and sSOI (2 and 4) nFinFETs of $W_{\text {eff }}=0.02 \mu \mathrm{m}$ (1 and 2) and $9.87 \mu \mathrm{m}$ (3 and 4) and at various $W_{\text {eff }}$ (b) for SOI (1) and sSOI (2) nFinFETs of $L_{\text {eff }}=0.9 \mu \mathrm{m} ; V_{\mathrm{GB}}=0$.

It should be noted that the higher values of $\mu_{m}$ in FinFETs where $W_{\text {eff }}=0.02 \mu \mathrm{m}<<2 h$ than in FinFETs where $W_{\text {eff }}=9.87 \mathrm{~m}>>2 h$ (Fig. $5 \mathrm{a}$ ) can be explained by $\mu_{\text {side }}>\mu_{\text {top }}$ [6], where $\mu_{\text {side }}$ and $\mu_{\text {top }}$ are the values of $\mu_{m}$ for the sidewall and top portions of the channel. However, it has been found that $\mu_{\text {side }}<\mu_{\text {top }}$ for nFinFETs [7]. Moreover, the method proposed in [6] can be used only in the case where $\mu \neq \mu\left(W_{\text {eff }}\right)$. At the same time, Fig. $5 \mathrm{~b}$ demonstrates the increase of $\mu_{\mathrm{m}}$ with decreasing $W_{\text {eff }}$ at $W_{\text {eff }}>0.9 \mu \mathrm{m}$ for the FinFETs, where $W_{\text {eff }}>>2 h$ and, hence, $\mu_{m}=\mu_{\text {top }}$. Therefore, like for the $65 \mathrm{~nm}$ FD planar SOI nMOSFETs [4], the increase of $\mu_{\text {top }}$ with decreasing $W_{\text {eff }}$ takes place in the FinFETs considered. Then such an increase of $\mu_{\text {top }}$ at $W_{\text {eff }}<0.9 \mu \mathrm{m}$ could be responsible for the higher values of $\mu_{m}$ in the FinFETs of $W_{\text {eff }}=0.02 \mu \mathrm{m}$ even under conditions where $\mu_{\text {side }}<\mu_{\text {top }}$.

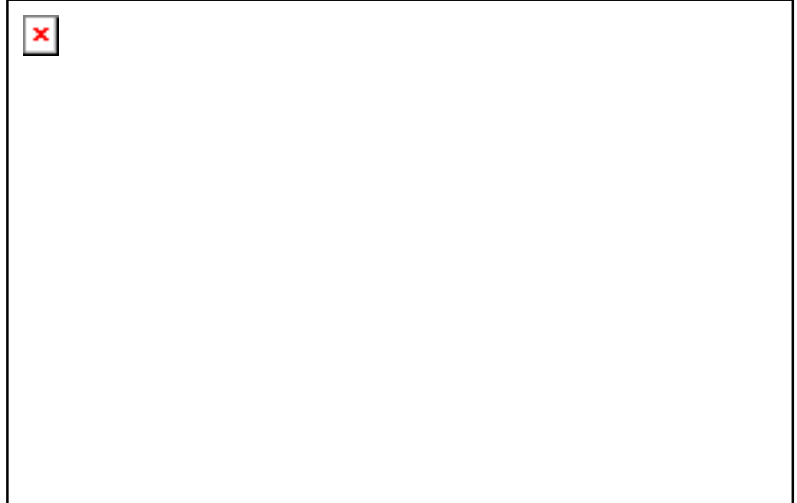

Fig. 6. Dependences of the drain current normalized for $L_{\mathrm{eff}}$ and $Z$ on the gate overdrive voltage for sSOI FinFETs of $L_{\text {eff }}=0.9 \mu \mathrm{m}$ and $W_{\text {eff }}=0.02 \mu \mathrm{m}(1)$ and $9.87 \mu \mathrm{m}(2)$ measured at $V_{\mathrm{GB}}=0$.

As to the behavior of $I$ with $V^{*}$, it has been found that the increase of I with increasing $V^{*}$ becomes sublinear at $V^{*} \geq 0.4 \mathrm{~V}$ (Fig. 6) that is at rather low $V^{*}$ where such an effect cannot be related to the influence of the series resistance and usually is attributed to the decrease of the electron mobility with increasing $V^{*}$. At the same time, our noise measurements have shown that $S_{I} \neq S_{I}\left(V^{*}\right)$ at $0.1 \mathrm{~V} \leq V^{*} \leq 0.8 \mathrm{~V}$ (Fig. 2). Since for the McWhorter noise $S_{I} \sim \mu^{2}$, this suggests that $\mu \neq \mu\left(V^{*}\right)$ in the above mentioned range of $V^{*}$ and, hence, the sublinear behavior of $I\left(V^{*}\right)$ at $V^{*} \geq 0.4 \mathrm{~V}$ is not explained by a decrease of $\mu$. The possible reason for this effect is the sublinear increase of the free electron density in the channel $N_{S}$ with increasing $V^{*}$. A similar situation has been observed previously for the planar nMOSFETs [2].

3. It has been found that under conditions where an accumulation voltage is applied to the back gate, the LKE Lorentzians [8] appear in the noise spectra measured at $1 \mathrm{~V} \leq V_{\mathrm{GF}} \leq 1.6 \mathrm{~V}$ for the sSOI and SOI nFinFETs of $W_{\text {eff }} \geq 0.9 \mu \mathrm{m}$ (Fig. 7). It should be noted that this is the first observation of the LKE noise under a back-gate accumulation bias for sufficiently wide nMuGFETs. The behavior of the parameters of those LKE Lorentzians (the Lorentzian plateau $\left[S_{I}(0)\right]_{\text {LKE }}$ and time constant $\left.\tau_{\mathrm{LKE}}\right)$ are shown in Fig. 8. It is seen from Fig. 8a that $\left[S_{I}(0)\right]_{\mathrm{LKE}} \sim \tau_{\mathrm{LKE}}$ that is typical for the LKE Lorentzians [8] and that $\left\{\left[S_{I}(0)\right]_{\mathrm{LKE}} / \tau_{\mathrm{LKE}}\right\} \sim\left(L_{\mathrm{eff}}\right)^{-n}$ where $n<3$ while $n=3$ has been observed for planar MOSFETs with similar lengths as in Fig. 8a [8]. Since $\left[S_{I}(0)\right]_{\mathrm{LKE}} \sim \mu^{2}[8]$, one of the reasons for this effect is the decrease of $\mu$ with decreasing $L_{\text {eff }}$.

As to the behavior of $\tau_{\mathrm{LKE}}$ with $V_{\mathrm{GF}}$, it is seen from Fig. 8b that: (i) the sSOI FinFETs are characterized by much higher values of $\tau_{\mathrm{LKE}}$ at one and the same values of $V_{\mathrm{GF}}$; (ii) $\tau_{\mathrm{LKE}} \neq \tau_{\mathrm{LKE}}\left(L_{\text {eff }}\right)$ at $L_{\text {eff }} \geq 0.9 \mu \mathrm{m}$ while the values of $\tau_{\mathrm{LKE}}$ for $L_{\mathrm{eff}}=0.4 \mu \mathrm{m}$ appear to be lower than for $L_{\text {eff }} \geq 0.9 \mu \mathrm{m}$, and this effect is more strong for the sSOI FinFETs than for the SOI ones. Since the considered LKE Lorentzians have been observed at 


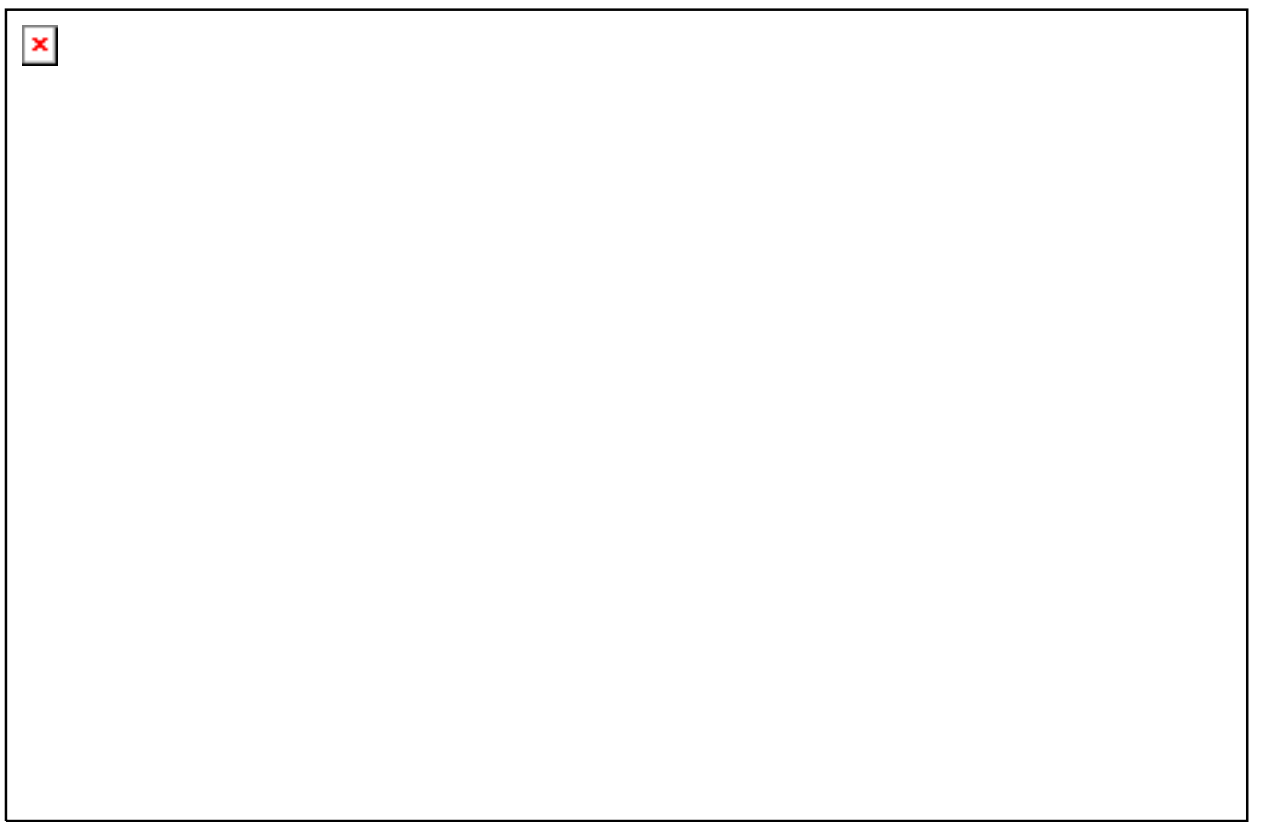

Fig. 7. Spectra of the drain current noise measured at accumulation back-gate voltage $V_{\mathrm{GB}}=-9.48 \mathrm{~V}$ for the SOI (a) and sSOI (b) FD nFinFETs of $W_{\text {eff }}=9.87 \mu \mathrm{m}$ and $L_{\text {eff }}=0.9 \mu \mathrm{m}$.

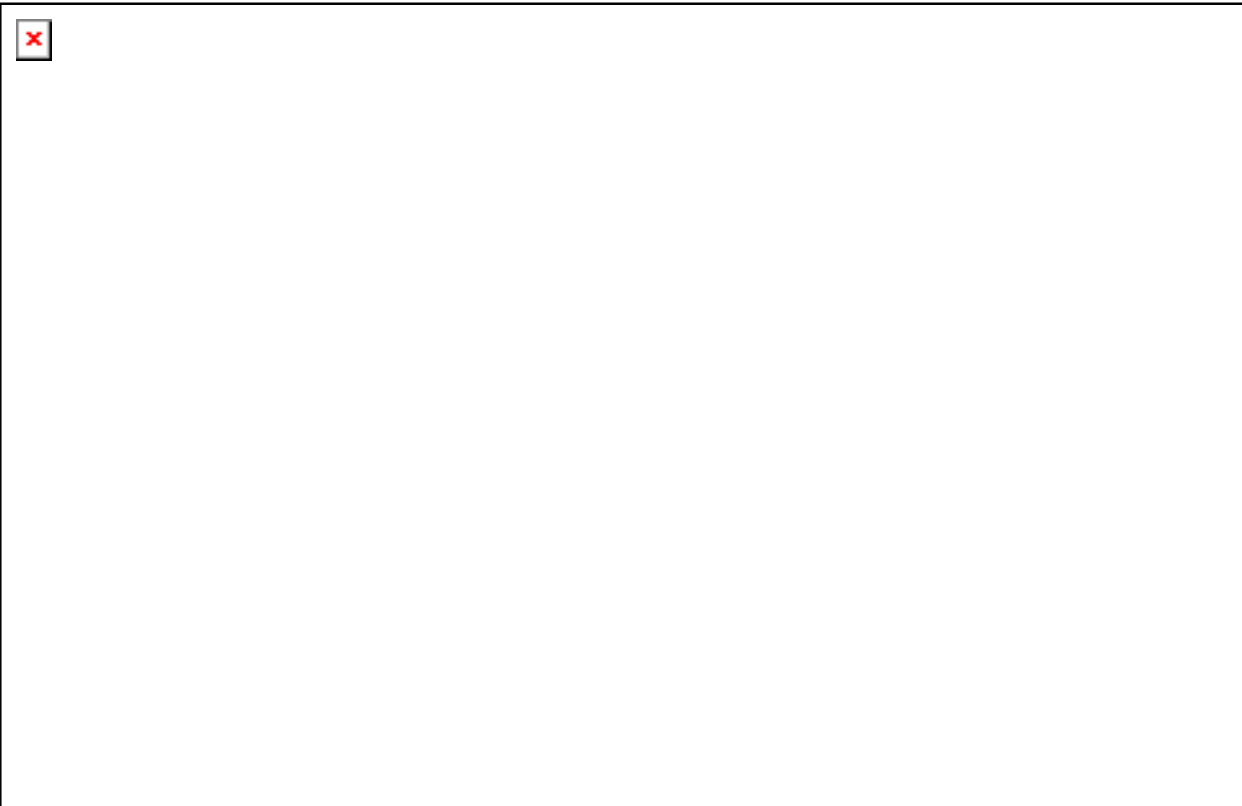

Fig. 8. Dependences of the LKE Lorentzian plateau on the time constant (a) and of the LKE Lorentzian time constant on the front gate voltage (b) measured at $V_{\mathrm{GB}}=-9.48 \mathrm{~V}$ for sSOI nFinFETs of $W_{\text {eff }}=9.87 \mu \mathrm{m}$ and $L_{\text {eff }}=2.9 \mu \mathrm{m}$ (1), 0.9 (2), and 0.4 (3).

$V^{*}<1 \mathrm{~V}$, where the values of $\tau_{\mathrm{LKE}}$ are determined by the electron valence band tunneling not only through the gate oxide but also through the silicon film depletion layer [9], this effect can be related with different conditions for such tunneling in sSOI and SOI FinFETs.

\section{Conclusions}

1. The low-frequency noise of the McWhorter type is typical at $V_{\mathrm{GB}}=0$ for the nFinFETs investigated. For the sSOI devices, the densities of the noisy traps located in the gate oxide at $x<1.5 \mathrm{~nm}$ and $x>1.5 \mathrm{~nm}$, where $x$ is the distance from the $\mathrm{Si} / \mathrm{SiO}_{2}$ interface into the oxide, are found to be $N_{\mathrm{ot}}=$ (2 to 3$) \times 10^{19} \mathrm{~cm}^{-3} \mathrm{eV}^{-1}$ and $N_{\mathrm{ot}}=(4$ to 8$) \times 10^{18} \mathrm{~cm}^{-3} \mathrm{eV}^{-1}$, respectively, the values of $N_{\text {ot }}$ are practically independent of $W_{\text {eff }}$ and $L_{\text {eff }}$; for the SOI ones of $W_{\text {eff }}=0.02 \mu \mathrm{m}$, the values of $N_{\text {ot }}$ appear to be lower.

2. The LKE Lorentzians have been revealed in the low-frequency noise spectra for the devices of $W_{\text {eff }} \geq 0.9 \mu \mathrm{m}$ at $1 \mathrm{~V} \leq V_{\mathrm{GF}} \leq 1.6 \mathrm{~V}$ when measuring at an 
accumulation back-gate voltage, and the parameters of those Lorentzians are found to be different for the sSOI and SOI nFinFETs.

3. The results obtained for the McWhorter noise suggest that: (i) the dependences of $\mu_{m}$ and $N_{\mathrm{ot}}$ on the device dimensions do not correlate; (ii) the reason for the sublinear increase of $I$ with increasing $V_{\mathrm{GF}}$ observed for the nFinFETs at $V^{*} \geq 0.4 \mathrm{~V}$ is the sublinear increase of $N_{S}$ but not the decrease of the electron mobility.

\section{References}

1. E. Simoen and C. Claeys, On the flicker noise in submicron silicon MOSFETs // Solid-State Electronics 43(5), p. 865-882 (1999).

2. N. Lukyanchikova, N. Garbar, V. Kudina, A. Smolanka, M. Lokshin, E. Simoen and C. Claeys, High gate voltage drain current leveling off and its low-frequency noise in $65 \mathrm{~nm}$ fully-depleted strained and non-strained SOI nMOSFETs // SolidState Electronics 52(5), p. 801-807 (2008).

3. R. Jayaraman and C. Sodini, A $1 / f$ technique to extract the oxide trap density near the conduction band edge of silicon // IEEE Trans. Electron Devices 36(9), p. 1773-1782 (1989).

4. N. Lukyanchikova, N. Garbar, V. Kudina, A. Smolanka, E. Simoen and C. Claeys, Behavior of the $1 / f$ noise and electron mobility in $65 \mathrm{~nm}$ FD SOI nMOSFETs employing different tensile-straininducing techniques, In: $19^{\text {th }}$ International Conference on Noise and Fluctuations - ICNF 2007, Eds. M. Takano, Y. Yamamoto, M. Nakao, p. 39-42, American Institute of Physics (2007).
5. J. Ramos, E. Augendre, A. Kottantharayil, A. Mercha, E. Simoen, M. Rosmeulen, S. Severi, C. Kerner, T. Chiarella, A. Nackaerts, I. Ferain, T. Hoffmann, M. Jurczak and S. Biesemans, Expderimental evidence of short-channel electron mobility degradation caused by interface charges located at the gate-edge of Triple-Gate FinFETs, In: Proc $8^{\text {th }}$ ICSICT, p. 72-74, Shanghai, China (2006).

6. V. Iyengar, A. Kottantharayil, F. Tranjan, M. Jurczak and K. De Meyer, Extraction of the top and sidewall mobility in FinFETs and the impact of Fin-patterning processes and gate dielectrics on mobility // IEEE Trans. Electron Devices 54(5), p. 1177-1184 (2007).

7. B. Yu, L. Chang, S. Ahmed, H. Wang, S. Bell, C.-Y. Yang, C. Tebery, C. Ho, Q. Xiang, T.-J. King, J. Bokor, C. Hu, M.-R.Lin and D. Kyser, FinFET scalling to $10 \mathrm{~nm}$ gate length, In: IEDM Tech. Dig., p. 251-254 (2002).

8. N. Lukyanchikova, M. Petrichuk, N. Garbar, A. Mercha, E. Simoen and C. Claeys, Electron valence-band tunneling-induced Lorentzian noise in deep submicron silicon-on-insulator metaloxide-semiconductor field-effect transistors // $\mathrm{J}$. Appl. Phys. 94, p. 4461-4469 (2003).

9. N. Lukyanchikova, Noise research of nanoscaled SOI devices, In: Nanoscaled Semiconductor-onInsulator Structures and Devices, Eds. S. Hall, A. Nazarov and V. Lysenko. Springer, 2007, p. 181198. 